\title{
On the Use of Edge Features and Exponential Decaying Number of Nodes in the Hidden Layers for Handwritten Signature Recognition
}

\author{
Teddy Surya Gunawan ${ }^{1}$, Mira Kartiwi ${ }^{2}$ \\ ${ }^{1}$ Electrical and Computer Engineering Department, International Islamic University Malaysia, Malaysia \\ ${ }^{2}$ Information Systems Department, International Islamic University Malaysia \\ 53100 Jalan Gombak, Kuala Lumpur, Malaysia
}

\begin{tabular}{l}
\hline \hline Article Info \\
\hline Article history: \\
Received Jun 4, 2018 \\
Revised Aug 2, 2018 \\
Accepted Aug 15, 2018 \\
\hline
\end{tabular}

\section{Keywords:}

Deep feedforward neural network

Edge detection

Exponential decaying

Hidden layers

Offline handwritten signature

\begin{abstract}
Handwritten signatures are playing an important role in finance, banking and education and more because it is considered the "seal of approval" and remains the most preferred means of authentication. In this paper, an offline handwritten signature authentication algorithm is proposed using the edge features and deep feedforward neural network (DFNN). The number of hidden layers in DFNN is configured to be at least one layer and more. In this paper, an exponential decaying number of nodes in the hidden layers was proposed to achieve better recognition rate with reasonable training time. Of the six edge algorithms evaluated, Roberts operator and Canny edge detectors were found to produce better recognition rate. Results showed that the proposed exponential decaying number of nodes in the hidden layers outperform other structure. However, more training data was required so that the proposed DFNN structure could have more efficient learning.
\end{abstract}

Copyright () 2018 Institute of Advanced Engineering and Science. All rights reserved.

\section{Corresponding Author:}

Teddy Surya Gunawan

Electrical and Computer Engineering Department, International Islamic University Malaysia, Malaysia.

Email: tsgunawan@iium.edu.my

\section{INTRODUCTION}

The handwritten signature is very important for every individual as it is widely used for authentication purposes in every day life. Each of the signers has their own signature, which is although unique but it could vary from time to time, or due to different tools used, such as pen with different pen size, stylus, or finger. The handwritten signature authentication system aims to minimize the intrapersonal differences [1]-[2]. Signature verification can be classified into two parts which is online and offline. This paper focuses on the offline system which could be considered as more challenging compare to the online system. This is due to the offline system did not capture the dynamic which can help the classifier to authenticate better the signatory [3].

Many researches have been conducted to develop handwritten signature authentication system. Typical handwritten signature authentication system is shown in Figure 1. In [4], an orientation of the skeleton and gravity center point were combined to extract more accurate features. Another feature, i.e. Euler number, was calculated as the subtraction of total number of objects in the handwritten image with total number of holes [5]. Other features has been as well in the literature, such as roundness, skewness, kurtosis, mean, standard deviation, area, distribution density, entropy, connected component and perimeter [2], [5]. 


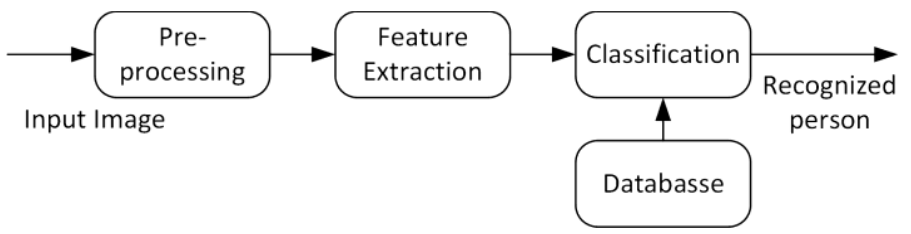

Figure 1. Typical handwritten signature authentication system

On the classifier part, two methods have been mostly utilized, i.e. SVM [6], neural network [2], [7]-[8], as well as deep neural network [1]. Although many researches have been conducted on offline handwritten signature authentication, but there are still many aspects have not been considered. In this paper, we have collected our own handwritten signature image database with variation in the position and pen size and color used to sign. Furthermore, our previous research showed that the use of high pass filter produce better accuracy compared to low pass filter [9]. The high pass filter used in [9] is only Canny edge detector. Therefore, in this paper, around six edge detection algorithms were used, including Sobel, Prewitt, Roberts, Laplacian of Gaussian, Zero Cross, and Canny.

Deep neural networks uses at least two hidden layers or more in their configuration [10]. The optimal structure of the neural networks is still an active research area. In [11], pruning method were used to obtain optimum DNN structure. However, the steps involved is rather complex. Therefore, the objective of this paper is to develop an offline handwritten signature using edge features and exponential decaying number of nodes in the hidden layers of DNN structure. The proposed algorithms will be evaluated in terms of training time and recognition rate.

\section{PROPOSED HANDWRITTE SIGNATURES USING DEEP FEEDFORWARD NEURAL NETWORKS}

Figure 2 shows our proposed algorithm, in which the system has two main parts, i.e. feature extraction and classifier. The feature extraction part is including the image segmentation, aligning and cropping, color to grayscale conversion, and image filtering. The classifier part is including the training and testing of neural network with the developed image database.

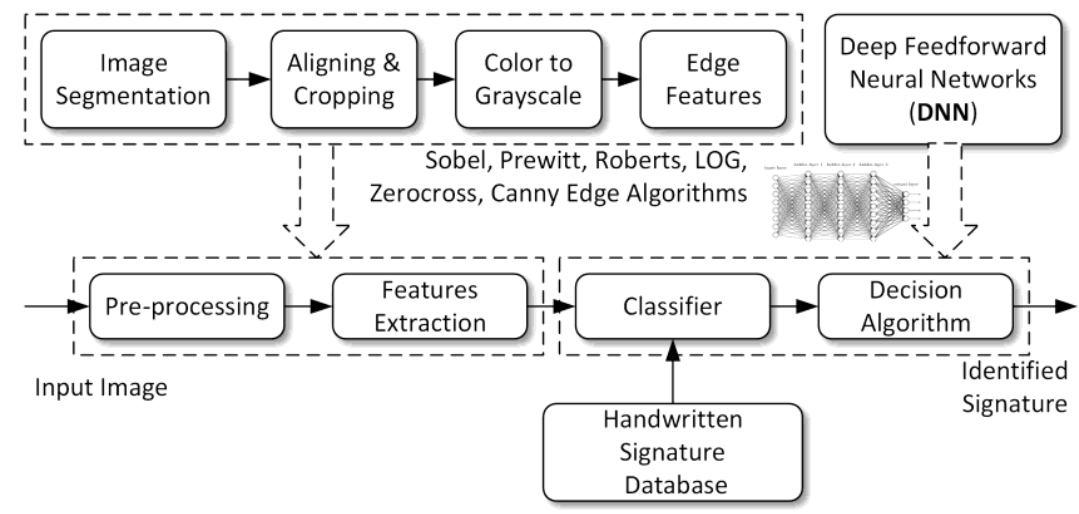

Figure 2. Proposed handwritten signature authentication system using deep neural networks

\subsection{Handwritte Signature Image Database And Edge Features}

The handwritten signatures are collected from five persons using five different pen size and styles, each for 10 ten times producing a total of 50 signatures have been taken for each person. The total handwritten signatures collected are 250 images [9]. The handwritten signatures are then scanned using a scanner to convert it to the digital images. The color image is converted to grayscale image to reduce the computation. The image size is fixed to be 206 by 128 pixels, which is then changed into column vector of 26368. Finally, the handwritten signatures database will be divided randomly for each person to be $50 \%$ for training, $10 \%$ for cross validation, and $40 \%$ for testing. Figure 3 shows the example of the collected signature images. 


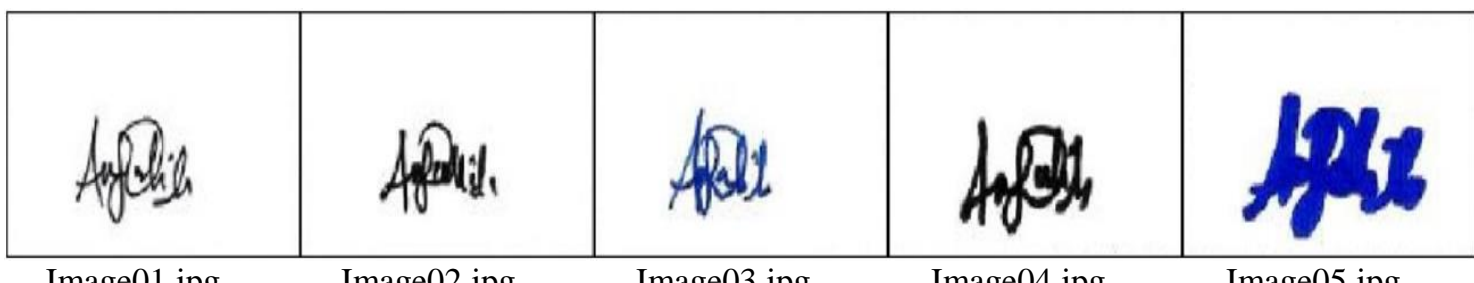

\begin{tabular}{llll|l} 
Image01.jpg $\quad$ Image02.jpg & Image03.jpg & Image04.jpg & Image05.jpg
\end{tabular}

Figure 3. Example of PersonA signatures with five different pen size and color

Our previous research showed that high pass filter produce better accuracy compared to low pass filter [9]. Although canny edge detection is the most popular method for edge detection [12], but we will evaluate its effectiveness in DNN configuration against five other algorithms. Figure 4 shows the example of edge detection algorithms for Image 02 and Image 05 of Person A. The different pen size and styles will produce different edge features, in which the similarity is rather low. In that case, the training of neural network will be rather difficult to achieve high recognition rate. Moreover, Table 1 shows the processing time of various edge detection algorithms to obtain edge features for all 250 images. It can be found that Prewitt operator is the fastest, while Canny edge detection is the slowest.

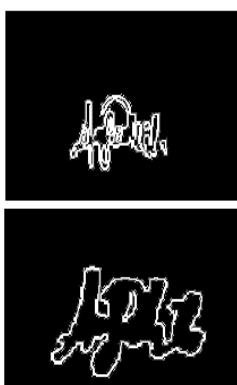

(a)

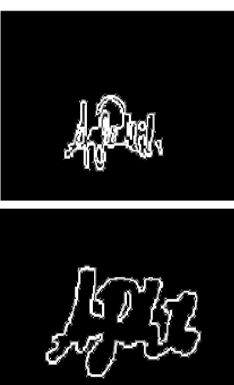

(b)

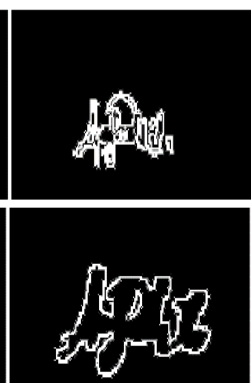

(c)

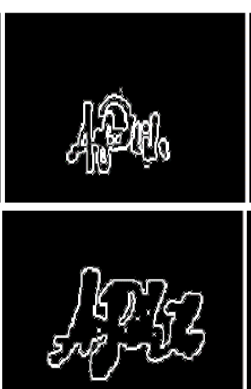

(d)

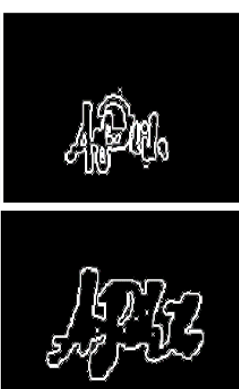

(e)

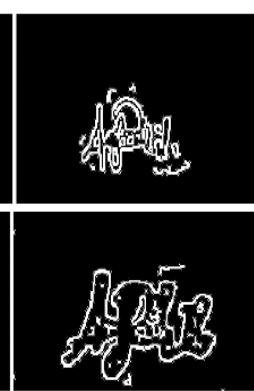

(f)

Figure 4. Example of persona signatures, i.e. image02.jpg (first row) and image05.jpg (second row), with different edge detection algorithms, (a) sobel, (b) prewitt, (c) roberts,

(d) laplacian of gaussian, (e) zero cross, (f) canny.

Table 1. Processing Time of Various Edge Detection Algorithms

\begin{tabular}{cc}
\hline Edge Features & Processing Time (seconds) \\
\hline Sobel & 0.7352 \\
Prewitt & 0.6048 \\
Roberts & 0.7808 \\
Laplacian of Gaussian & 0.8991 \\
ZeroCross & 1.0468 \\
Canny & 1.1808 \\
\hline
\end{tabular}

\subsection{Exponential Decaying Number of Nodes in the Hidden Layers}

The main principle of DNN is to utilize lower level features learning to update the learning of higher features. There are many available deep architecture, such as neural networks with many hidden layers and/or many hidden variables, convolutional neural networks, recurrent neural networks, and deep belief network [10], [13]. In this research, we used deep learning using feedforward neural network architectures with hidden layers with many hidden variables [14]. Figure 5 illustrates the deep feedforward neural network structure. 


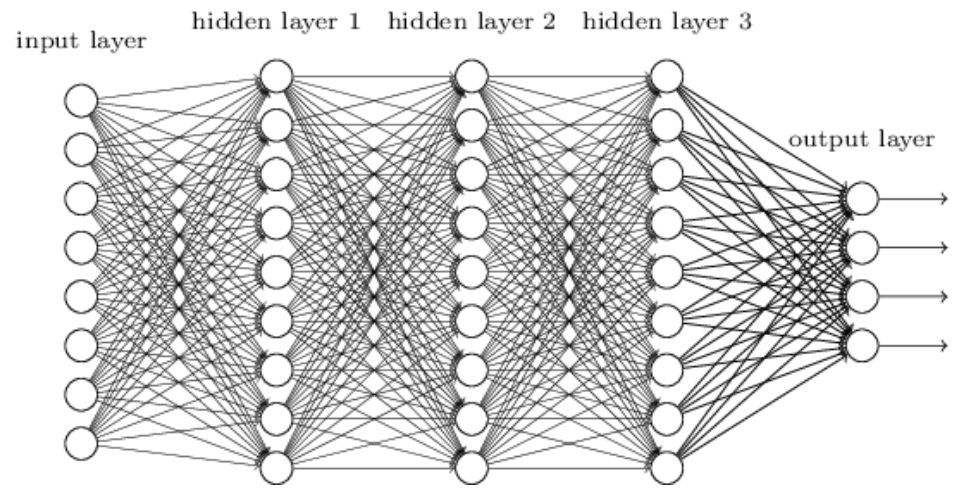

Figure 5. Deep feedforward neural network structure

In this paper, we propose an exponential decaying number of hidden nodes due to its simplicity compared to pruning method as described in [11]. It can be formulated as follows:

$$
y=a \cdot e^{-b x}
$$

Where $x$ is neural network layers, while $y$ is the number of nodes. Input layer is defined as $x=0$, while the last layer will be the output layer. For example, if we have 3 hidden layers (NHidden $=3$ ), $x=0$ is the input layer, $x=1$ is the first hidden layer, $x=2$ is the second hidden layer, $x=3$ is the third hidden layer, and $x=4$ is the output layer. Using curve fitting, we could find the parameter $a$ and $b$. In our case, at $x=0$ parameter $a=26368$ (input layer) and at $x=4 \rightarrow y=5$ (output layer). Then, parameter $b$ could be calculated as follows:

$$
b=-\frac{1}{\text { NHidden }} \log \left(\frac{\text { OutputLayer }}{\text { InputLayer }}\right)
$$

Where NHidden is the number of hidden layers, InputLayer is the input nodes, and OutputLayer is the output nodes. Table 2 shows the example of exponential decaying number of nodes in various hidden layers, when the InputLayer $=26368$ and OutputLayer $=5$, using Eq. (1) and (2). Figure 6 illustrates the exponential decaying number of nodes for 4 hidden layers.

Table 2. Exponential Decaying Number of Nodes in Various Hidden Layers Structures

\begin{tabular}{cc}
\hline NHidden & Hidden Layer Nodes Configuration \\
\hline 1 & {$[363]$} \\
2 & {$[151587]$} \\
3 & {$[309436343]$} \\
4 & {$[475085615428]$} \\
5 & {$[632015153638721]$} \\
6 & {$[775122786701975817]$} \\
\hline
\end{tabular}

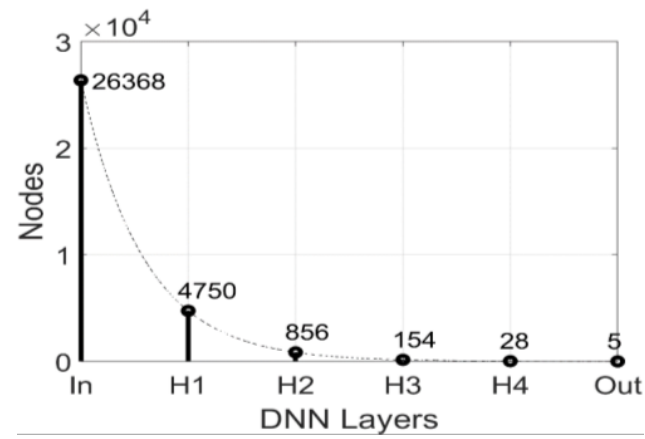

Figure 6. Example of exponential decaying number of nodes for NHidden $=4$ 


\section{RESULTS AND ANALYSIS}

In this section, the experimental setup, handwritten signature image database and its edge features, training and testing of deep feedforward neural network with exponential decaying number of nodes in the hidden layers will be discussed.

\subsection{Experimental Setup}

A high performance system was used for processing, i.e. a multicore system with Intel Core i7 6700 K 4.00 GHz (4 cores with 8 threads), 32 GBytes RAM, 256 GBytes SSD and 2 TBytes hard disk, installed with Windows 10 operating system and Matlab 2018a with Image Processing, Signal Processing and Neural Network Toolboxes. The handwritten signature database were collected from five person, hence the output layer is set to five. The number of nodes in the hidden layer as well as hidden layer will be varied, while the patternet() Matlab function will be used. Other types of feedforward neural network can be used as well, as described in [14].

\subsection{Training Phase of Various DFNN Structures}

In Table 3, the best recognition rates were highlighted in bold. Across the row, the maximum recognition rate is achieved when the structure of hidden layers are [1000 100 10] and [3094 363 43] with recognition rate of $96.89 \%$ and $96.67 \%$, respectively. While across the column, the maximum recognition rate is achieved for Robert and Canny edge detection algorithms with recognition rate of $89.72 \%$ and $88.33 \%$, respectively. Therefore, these two edge features and two DFNN structures will be further trained and tested with the new images. Moreover, the last six rows of Table 3 shows the performance of the exponential decaying number of nodes for hidden layer of one to six. Compared to the other DFNN structures, it consistently shows high average recognition rate across various edge features.

Table 3. Recognition Rate (\%) of Various DFNN Structures

\begin{tabular}{|c|c|c|c|c|c|c|c|}
\hline NHidden & Sobel & Prewitt & Roberts & LoG & zerocross & Canny & Average \\
\hline [10] & 96.00 & 96.67 & 96.67 & 86.67 & 96.00 & 95.33 & 94.56 \\
\hline [20] & 74.00 & 93.33 & 97.33 & 91.33 & 92.67 & 98.67 & 91.22 \\
\hline [30] & 96.00 & 98.00 & 97.33 & 94.00 & 96.67 & 94.00 & 96.00 \\
\hline [40] & 96.67 & 97.33 & 94.00 & 96.00 & 94.67 & 96.00 & 95.78 \\
\hline$[50]$ & 96.67 & 94.67 & 96.00 & 96.00 & 96.00 & 95.33 & 95.78 \\
\hline [100] & 96.00 & 96.67 & 94.67 & 91.33 & 93.33 & 95.33 & 94.56 \\
\hline [200] & 97.33 & 86.00 & 84.00 & 95.33 & 75.33 & 96.67 & 89.11 \\
\hline [300] & 94.00 & 86.67 & 95.33 & 86.67 & 95.33 & 96.00 & 92.33 \\
\hline [400] & 96.00 & 95.33 & 93.33 & 95.33 & 94.67 & 76.67 & 91.89 \\
\hline [500] & 94.67 & 84.67 & 95.33 & 94.00 & 95.33 & 94.00 & 93.00 \\
\hline [1000] & 93.33 & 94.00 & 94.00 & 82.00 & 92.67 & 20.00 & 79.33 \\
\hline [2000] & 95.33 & 92.67 & 69.33 & 93.33 & 93.33 & 95.33 & 89.89 \\
\hline [3000] & 94.67 & 60.00 & 90.67 & 88.00 & 88.67 & 93.33 & 85.89 \\
\hline [4000] & 90.00 & 23.33 & 91.33 & 74.67 & 92.67 & 81.33 & 75.56 \\
\hline$[5000]$ & 57.33 & 93.33 & 77.33 & 89.33 & 92.67 & 70.67 & 80.11 \\
\hline [10000] & 58.67 & 20.00 & 56.67 & 72.67 & 69.33 & 42.00 & 53.22 \\
\hline$\left[\begin{array}{ll}10 & 10\end{array}\right]$ & 73.33 & 72.00 & 78.67 & 71.33 & 20.00 & 84.67 & 66.67 \\
\hline$\left[\begin{array}{ll}20 & 20\end{array}\right]$ & 92.67 & 23.33 & 91.33 & 94.00 & 92.00 & 98.00 & 81.89 \\
\hline$\left[\begin{array}{ll}30 & 30\end{array}\right]$ & 81.33 & 95.33 & 95.33 & 22.67 & 94.67 & 96.67 & 81.00 \\
\hline$\left[\begin{array}{ll}40 & 40\end{array}\right]$ & 96.67 & 94.00 & 64.67 & 95.33 & 94.67 & 96.00 & 90.22 \\
\hline$\left[\begin{array}{ll}50 & 50]\end{array}\right]$ & 97.33 & 95.33 & 95.33 & 56.67 & 50.67 & 94.67 & 81.67 \\
\hline$\left[\begin{array}{ll}100 & 100\end{array}\right]$ & 30.67 & 96.67 & 92.67 & 92.00 & 43.33 & 95.33 & 75.11 \\
\hline$\left[\begin{array}{ll}100 & 10\end{array}\right]$ & 91.33 & 95.33 & 90.67 & 96.00 & 92.00 & 78.67 & 90.67 \\
\hline$\left[\begin{array}{lll}10 & 10 & 10\end{array}\right]$ & 66.67 & 20.00 & 78.00 & 84.00 & 67.33 & 50.67 & 61.11 \\
\hline$\left[\begin{array}{lll}20 & 20 & 20\end{array}\right]$ & 80.67 & 92.00 & 74.67 & 95.33 & 94.00 & 80.67 & 86.22 \\
\hline$\left[\begin{array}{llll}30 & 30 & 30\end{array}\right]$ & 96.00 & 96.67 & 77.33 & 87.33 & 34.67 & 96.00 & 81.33 \\
\hline$\left[\begin{array}{llll}40 & 40 & 40\end{array}\right]$ & 95.33 & 94.67 & 94.00 & 96.00 & 69.33 & 96.00 & 90.89 \\
\hline$\left[\begin{array}{lll}50 & 50 & 50\end{array}\right]$ & 96.00 & 95.33 & 96.67 & 94.00 & 94.67 & 97.33 & 95.67 \\
\hline$\left[\begin{array}{llll}100 & 100 & 100\end{array}\right]$ & 94.67 & 96.00 & 98.00 & 95.33 & 54.00 & 95.33 & 88.89 \\
\hline$\left[\begin{array}{llll}1000 & 100 & 10\end{array}\right]$ & 96.67 & 96.00 & 98.67 & 96.67 & 96.00 & 97.33 & 96.89 \\
\hline [363] & 94.67 & 94.67 & 94.00 & 94.67 & 94.00 & 96.00 & 94.67 \\
\hline [1515 87] & 84.67 & 90.67 & 95.33 & 94.67 & 96.67 & 98.00 & 93.34 \\
\hline [3094 363 43] & 96.67 & 98.00 & 98.00 & 95.33 & 95.33 & 96.67 & 96.67 \\
\hline$\left[\begin{array}{lllll}4750 & 856 & 154 & 28\end{array}\right]$ & 94.00 & 96.00 & 98.00 & 95.33 & 95.33 & 96.67 & 95.89 \\
\hline$\left[\begin{array}{llllll}6320 & 1515 & 363 & 87 & 21\end{array}\right]$ & 95.33 & 95.33 & 98.00 & 96.00 & 97.33 & 96.67 & 96.44 \\
\hline$\left[\begin{array}{llllll}7751 & 2278 & 670 & 197 & 58 & 17\end{array}\right]$ & 70.00 & 96.67 & 97.33 & 96.67 & 96.00 & 98 & 92.45 \\
\hline Average & 87.54 & 84.63 & 89.72 & 88.22 & 84.20 & 88.33 & 87.11 \\
\hline
\end{tabular}


In the training phase, $50 \%$ of the image database was used for training, and $10 \%$ was used for cross validation. The activaton function used on each nodes in the hidden layers is sigmoid function, except for the output layer which is softmax layer. The scaled conjugate gradient was used as the training function of backpropagation algorithm. The DFNN were trained for 50 epochs. For each row in Table 3, the number of nodes are the same, therefore the training time is relatively similar.

\subsection{Optimization and Testing Phase of Various DFNN Structures}

For testing purpose, we set the number of epochs to 1000 . Table 4 shows the results of the training and testing of the optimum DFNN structures for two edge features, i.e. Roberts and Canny algorithms. On average, the proposed exponential decaying number of nodes DFNN strucutures performed better in terms of testing recognition rate across two edge features, i.e. $69 \%$ compared to $66 \%$. Based on this result, another experiments using exponential decaying DFNN structures was conducted using Canny edge features only and the results is presented in Table 5. For better generalization and to avoid over-fitting, the training epoch was set to 200 as the maximum performance in cross validation is achieved at much lower epoch then 1000 .

Table 4. Training and Testing of Optimum DFNN Structures

\begin{tabular}{ccccc}
\hline \multirow{2}{*}{ NHidden } & $\begin{array}{c}\text { Edge } \\
\text { Features }\end{array}$ & $\begin{array}{c}\text { Training } \\
\text { Time }(\mathrm{s})\end{array}$ & $\begin{array}{c}\text { Training Recognition Rate } \\
(\%)\end{array}$ & $\begin{array}{c}\text { Testing Recognition Rate } \\
(\%)\end{array}$ \\
\hline$\left[\begin{array}{llcl}1000 & 100 & 10\end{array}\right]$ & Roberts & 1718 & 95.33 & 65.00 \\
{$\left[\begin{array}{lll}1000 & 100 & 10\end{array}\right]$} & Canny & 2295 & 97.33 & 67.00 \\
{$[309436343$} & Roberts & 5144 & 98.00 & 68.00 \\
\hline
\end{tabular}

Table 5. Testing Recognition Rate of Exponential Decaying DFNN Structures

\begin{tabular}{cccc}
\hline NHidden & $\begin{array}{c}\text { Training Time } \\
(\mathrm{s})\end{array}$ & $\begin{array}{c}\text { Training Recognition Rate } \\
(\%)\end{array}$ & $\begin{array}{c}\text { Testing Recognition Rate } \\
(\%)\end{array}$ \\
\hline$[363]$ & 170 & 93.33 & 51.00 \\
{$[151587]$} & 690 & 96.67 & 69.00 \\
{$[309436343]$} & 1418 & 96.67 & 72.00 \\
{$[475085615428]$} & 2238 & 100.00 & 73.00 \\
{$[632015153638721]$} & 3108 & 99.33 & 75.00 \\
{$[775122786701975817]$} & 3966 & 96.00 & 64.00 \\
\hline
\end{tabular}

From Table 5, although the performance of a trained DFNN is non deterministic, it could be concluded that there is a positive trends in the recognition rate when the number of hidden layers of DFNN were increased from 1 to 5 . While increasing futher the number of layer did not improve the recognition rate. This could be cause by overfitting or less of training data to better use of the deeper layers. Interestingly, the training recognition rate of 100 percent did not translate into higher testing recognition rate. In summary, the proposed exponential decaying number of nodes in the hidden layers provide a better DFNN structure with high recognition rate. The proposed system could be evaluated with other handwritten image database to evaluate further its effectiveness.

\section{CONCLUSION}

This paper has presented offline handwritten signature authentication system using edge features and DFNN. We formulated exponential decaying number of nodes in the hidden layers of DFNN structures and used it on DFNN. Moreover, six edge detection algorithms were evaluated, in which it was found that Roberts and Canny edge operators producing higher performance. Handwritten image database has been recorded which consists of 10 trial with 5 different pen of 5 person producing total of 250 images. Results showed that our proposed method, i.e. exponential decaying number of nodes in the hidden layers, produce higher accuracy. The highest testing recognition rate was $75.0 \%$ using five hidden layers. Further research includes the use of different handwritten image database, or the use of other types of deep learning like convolutional neural networks. 


\section{ACKNOWLEDGMENTS}

The researchers in this study would like to acknowledge the International Islamic University Malaysia (IIUM) for the financial funding of this research through the Research Initiatives Grant Scheme (RIGS) RIGS15-070-0070.

\section{REFERENCES}

[1]. D. Beatrice and H. Thomas, "On-line Handwritten Signature Verification using Machine Learning Techniques with a Deep Learning Approach. Master's Theses in Math," Sciences, Lund University, 2015.

[2]. A. Abushariah, T. Gunawan, J. Chebil, and M. Abushariah, "Automatic person identification system using handwritten signatures," in Computer and Communication Engineering (ICCCE), 2012 International Conference on, pp. 560-565, 2012.

[3]. B. Nassi, A. Levy, Y. Elovici, and E. Shmueli, "Handwritten Signature Verification Using Hand-Worn Devices," arXiv preprint arXiv:1612.06305, 2016.

[4]. K. Neamah, D. Mohamad, T. Saba, and A. Rehman, "Discriminative features mining for offline handwritten signature verification," 3D Research, vol. 5, pp. 1-6, 2014.

[5]. P. Maji, S. Chatterjee, S. Chakraborty, N. Kausar, S. Samanta, and N. Dey, "Effect of Euler number as a feature in gender recognition system from offline handwritten signature using neural networks," in Computing for Sustainable Global Development (INDIACom), 2015 2nd International Conference on, pp. 1869-1873, 2015.

[6]. Y. Guerbai, Y. Chibani, and B. Hadjadji, "The effective use of the one-class SVM classifier for handwritten signature verification based on writer-independent parameters," Pattern Recognition, vol. 48, pp. 103-113, 2015.

[7]. A. Pansare and S. Bhatia, "Handwritten signature verification using neural network," International Journal of Applied Information Systems, vol. 1, pp. 44-49, 2012.

[8]. B. Erkmen, N. Kahraman, R. A. Vural, and T. Yildirim, "Conic section function neural network circuitry for offline signature recognition," IEEE transactions on neural networks, vol. 21, pp. 667-672, 2010.

[9]. T. S. Gunawan, N. Mahamud, and M. Kartiwi, "Development of offline handwritten signature authentication using artificial neural network," in Computing, Engineering, and Design (ICCED), 2017 International Conference on, pp. 1-4, 2017.

[10]. P. Kim, "MATLAB Deep Learning," With Machine Learning, Neural Networks and Artificial Intelligence, 2017.

[11]. P. Thomas and M.-C. Suhner, "A new multilayer perceptron pruning algorithm for classification and regression applications," Neural Processing Letters, vol. 42, pp. 437-458, 2015.

[12]. T. S. Gunawan, I. Z. Yaacob, M. Kartiwi, N. Ismail, N. F. Za'bah, and H. Mansor, "Artificial Neural Network Based Fast Edge Detection Algorithm for MRI Medical Images," Indonesian Journal of Electrical Engineering and Computer Science (IJEECS), vol. 7, pp. 123-130, 2017.

[13]. Y. LeCun, Y. Bengio, and G. Hinton, "Deep learning," Nature, vol. 521, pp. 436, 2015.

[14]. M. F. Alghifari, T. S. Gunawan, and M. Kartiwi, "Speech Emotion Recognition Using Deep Feedforward Neural Network," Indonesian Journal of Electrical Engineering and Computer Science (IJEECS), vol. 10, 2018.

\section{BIOGRAPHIES OF AUTHORS}

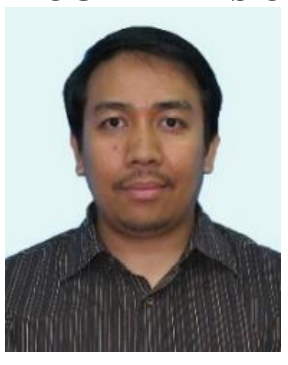

Teddy Surya Gunawan received his BEng degree in Electrical Engineering with cum laude award from Institut Teknologi Bandung (ITB), Indonesia in 1998. He obtained his M.Eng degree in 2001 from the School of Computer Engineering at Nanyang Technological University, Singapore, and $\mathrm{PhD}$ degree in 2007 from the School of Electrical Engineering and Telecommunications, The University of New South Wales, Australia. His research interests are in speech and audio processing, biomedical signal processing and instrumentation, image and video processing, and parallel computing. He is currently an IEEE Senior Member (since 2012), was chairman of IEEE Instrumentation and Measurement Society - Malaysia Section (2013 and 2014), Associate Professor (since 2012), Head of Department (2015-2016) at Department of Electrical and Computer Engineering, and Head of Programme Accreditation and Quality Assurance for Faculty of Engineering (since 2017), International Islamic University Malaysia. He is Chartered Engineer (IET, UK) and Insinyur Profesional Madya (PII, Indonesia) since 2016.

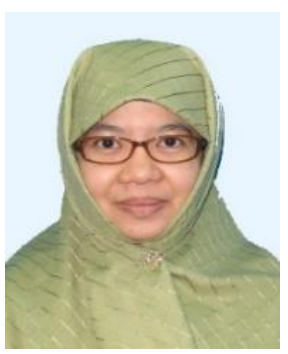

Mira Kartiwi completed her studies at the University of Wollongong, Australia resulting in the following degrees being conferred: Bachelor of Commerce in Business Information Systems, Master in Information Systems in 2001 and her Doctor of Philosophy in 2009. She is currently an Associate Professor in Department of Information Systems, Kuliyyah of Information and Communication Technology, International Islamic University Malaysia. Her research interests include electronic commerce, data mining, e-health and mobile applications development. 
